\title{
Feasibility assessment of invigorating grassrooTs primary healthcare for prevention and management of cardiometabolic diseases in resource- limited settings in China, Kenya, Nepal, Vietnam (the FAITH study): rationale and design
}

Enying Gong ${ }^{1}$, Hongsheng Lu' ${ }^{1}$, Shuai Shao ${ }^{1}$, Xuanchen Tao ${ }^{1}$, Nicholas Peoples ${ }^{1}$, Brandon A. Kohrt ${ }^{2,3}$, Shangzhi Xiong ${ }^{1}$, Catherine Kyobutungi ${ }^{4}$, Tilahun Nigatu Haregu ${ }^{4}$, Christopher Khayeka-Wandabwa ${ }^{4}$, Hoang Van Minh ${ }^{5}$, Tran Thi Duc Hanh ${ }^{5}$, Suraj Koirala ${ }^{6}$, Kamal Gautam ${ }^{6}$ and Lijing L. Yan ${ }^{1,2^{*}}$ (i)

\begin{abstract}
Background: Cardiometabolic diseases are the leading cause of death and disability in many low- and middleincome countries. As the already severe burden from these conditions continues to increase in low- and middleincome countries, cardiometabolic diseases introduce new and salient public health challenges to primary health care systems. In this mixed-method study, we aim to assess the capacity of grassroots primary health care facilities to deliver essential services for the prevention and control of cardiometabolic diseases. Built on this information, our goal is to propose evidence-based recommendations to promote a stronger primary health care system in resource-limited settings.
\end{abstract}

Methods: The study will be conducted in resource-limited settings in China, Kenya, Nepal, and Vietnam using a mixed-method approach that incorporates a literature review, surveys, and in-depth interviews. The literature, statistics, and document review will extract secondary data on the burden of cardiometabolic diseases in each country, the existing policies and interventions related to strengthening primary health care services, and improving care related to non-communicable disease prevention and control. We will also conduct primary data collection. In each country, ten grassroots primary health care facilities across representative urban-rural regions will be selected. Health care professionals and patients recruited from these facilities will be invited to participate in the facility assessment questionnaire and patients' survey. Stakeholders - including patients, health care professionals, policymakers at the local, regional, and national levels, and local authorities - will be invited to participate in indepth interviews. A standard protocol will be designed to allow for adaption and localization in data collection instruments and procedures within each country.

(Continued on next page)

\footnotetext{
* Correspondence: Lijing.yan@duke.edu

${ }^{1}$ Global Health Research Center, Duke Kunshan University, No. 8 Duke Avenue, Kunshan 215316, Jiangsu, China

${ }^{2}$ Global Health Institute, Duke University, Durham, NC, USA

Full list of author information is available at the end of the article
}

(c) The Author(s). 2019 Open Access This article is distributed under the terms of the Creative Commons Attribution 4.0 International License (http://creativecommons.org/licenses/by/4.0/), which permits unrestricted use, distribution, and reproduction in any medium, provided you give appropriate credit to the original author(s) and the source, provide a link to the Creative Commons license, and indicate if changes were made. The Creative Commons Public Domain Dedication waiver (http://creativecommons.org/publicdomain/zero/1.0/) applies to the data made available in this article, unless otherwise stated. 
(Continued from previous page)

Discussion: With a special focus on the capacity of primary health care facilities in resource-limited settings in lowand middle-income countries, this study has the potential to add new evidence for policymakers and academia by identifying the most common and significant barriers primary health care services face in managing and preventing cardiometabolic diseases. With these findings, we will generate evidence-based recommendations on potential strategies that are feasible for resource-limited settings in combating the increasing challenges of cardiometabolic diseases.

Keywords: Cardiometabolic diseases, Primary health care, Developing countries, Resource-limited settings

\section{Background}

Cardiometabolic diseases (CMDs), including both cardiovascular diseases and diabetes mellitus, are the leading cause of death and disability across the globe [1]. According to new estimates from the Global Burden of Disease study, cardiovascular diseases and diabetes mellitus resulted in 17.79 million and 1.37 million global deaths, respectively, in 2017, which had increased by 21.1 and $34.8 \%$ since 2007 [1]. Although previous research on CMDs has largely focused on high-income countries, CMDs have become an increasing challenge to most low- and middle-income countries (LMICs) where about $80 \%$ of non-communicable disease (NCD)related deaths occur. Exposure to risk factors for metabolic diseases has also increased in most LMICs in the past three decades [2]. CMDs further impose a substantial economic burden on LMICs: the anticipated economic burden of cardiovascular diseases in LMICs between 2011 and 2025 will be 3.76 trillion USD during the period of 2011 to 2025 [3]. This rapidly increasing burden of CMDs in LMICs needs immediate action in policies, health care services, and among the public.

Strengthening primary health care (PHC) is a key strategy for effective NCDs prevention and control [4-7]. Decades of evidence show that a strong PHC system rooted in the community can support the delivery of high-quality health care services at a lower cost than non-community based support and can respond to evolving health challenges $[8,9]$. In 2010, the World Health Organization published its "Package of Essential NCD interventions for primary care in low-resource settings" (WHO PEN) to provide a prioritized set of cost-effective interventions ("best buys") that are high-impact and feasible for implementation even in resource-constrained settings [10]. WHO PEN is a worthwhile approach for having managed NCDs, having progressed from its initial rollout in pilot sites of selected countries $[2,11]$ and showing promising results to becoming standard practice in countries such as Bhutan and Myanmar. For instance, in July 2017, the Prime Minister of Timor-Leste formally incorporated the PEN package into primary healthcare [12]. There is concern, however, about ensuring that the implementation of PEN interventions in matched with improving primary care facilities' capacity to deliver these services since previous studies have shown large gaps between their capacity and WHO PEN standards [13-16]. As countries vary in terms of health system structure, economic development, and policies for addressing NCDs, there is a need to understand the gaps between the capacity of PHC facilities and delivering essential services to people with CMDs, especially grassroots facilities in LMICs.

Previous studies have investigated the capacity of primary healthcare facilities in delivering healthcare services for NCDs prevention; however, most have only analyzed internal facility capacity through crosssectional surveys $[13,17,18]$. For example, one study conducted a cross-sectional survey of 90 PHC facilities in eight LMICs to measure the availability of factors such as human resources, equipment, and medicines [13]. While the major resource deficits were examined, the underlying reasons and related internal and external factors were not. Many commentary pieces have recommended that improving primary healthcare capacity in CMDs prevention and control needs political commitment and resource input, strong integration within the health system, connected care with other services, and awareness and acceptance from patients [19-21]. Therefore, we need to take a more comprehensive view to understand the capacity of primary healthcare facilities, especially with respect to interaction with patients and implementation of policies. In addition, we need to adopt mixed-methods to go beyond identifying key deficits and further understand the internal and external factors which influence capacity building and service delivery.

Another research gap is that most studies have focused on analyzing PHC facilities within specific healthcare systems [22, 23], while only a few have examined the common challenges and strategies that may be feasible regionally or across countries [13]. For example, grassroots PHC facilities may face difficulties in translating political commitment and national strategies into practice and have limited resources to invest in healthcare services. Thus, there is a need for cross-country studies to highlight the common challenges faced by grassroots PHC facilities and those strategies which may best 
facilitate PHC strengthening. By providing such a crosscountry comparison, this study will make evidence-based recommendations for strategies which can be feasibly and broadly adopted across resource-limited settings.

In light of these research gaps, we will conduct the present study in resource-limited areas in four countries: China, Nepal, Vietnam, and Kenya.

\section{Study design \\ Overview}

For this study, we will utilize multiple research methods, including literature reviews, quantitative surveys, and qualitative interviews to collect data. Specifically, the study will:

1) Review literature, statistics, and documents to synthesize existing information on the burden and trends of CMDs as well as national policies for CMDs prevention and control, with a focus on $\mathrm{PHC}$ services in resource-limited settings;

2) Conduct facility assessment surveys to evaluate the capacity of PHC facilities and to perform in-depth interviews with leaders of PHC facilities to identify gaps, barriers, and enabling and reinforcing factors in delivering healthcare services related to CMDs prevention and control;

3) Perform cross-sectional surveys and in-depth interviews with people with CMDs to gain a deeper understanding of factors that influence patients' accessibility and utilization of services, and

4) Conduct in-depth interviews among policymakers at national, regional and district level to understand the implementation of policy strategies and to identify factors that related to PHC system strengthening and NCDs related program implementation. Figure 1 provides an overview of the study design, the linkage of research activities, and the specific research question to be addressed.

The project protocol is developed by the core research team and will be implemented and adapted by researchers in four countries (China, Nepal, Vietnam, and Kenya) based on the local contexts. Research activities will be conducted at the national, regional, and facility level. All first-hand data collection will be conducted by the research team in the country, considering the familiarity with local languages, the country context, and the feasibility in study implementation. Table 1 shows the scope of fieldwork activities planned in each country.

\section{Study setting}

We will conduct this study in four countries: China, Kenya, Nepal and Vietnam. These four countries are similar in that they are all confronting the increasing burden of CMDs [24-27] and have plans to strengthen their primary healthcare systems to combat the increasing challenges of CMDs to the healthcare system. They are distinctive from each other, however, in demographic structure and socioeconomic development (see Table 2). The comparison of research findings across these four countries has the potential to demonstrate common gaps and challenges. In addition, the selection of these countries is based on funder requirements and our research collaboration network, which ensure the feasibility and quality in implementing the study protocol.

In each country, the local research team will identify two representative resource-limited regions to conduct the study. The two areas will be selected based on

\begin{tabular}{|c|c|c|c|}
\hline \multirow{3}{*}{ Review } & \multicolumn{3}{|c|}{ Literature, statistics and document review } \\
\hline & \multicolumn{3}{|c|}{$\begin{array}{l}\text { What is the burden and trend of } \mathrm{CMDs} \text { in the country? } \\
\text { What are the existing and planned national policies and strategies for } \mathrm{CMDs} \text { prevention and control that delivered } \\
\text { through primary healthcare facilities? }\end{array}$} \\
\hline & $\begin{array}{l}\text { Policymakers at national- } \\
\text { regional- and district-level }\end{array}$ & $\begin{array}{l}\text { Grassroot Primary } \\
\text { healthcare facilities }\end{array}$ & $\begin{array}{l}\text { People with cardio- } \\
\text { metabolic diseases }\end{array}$ \\
\hline Field activities & $\begin{array}{l}\text { In-depth interviews } \\
\text { implementation for CMDs } \\
\text { prevention and control at } \\
\text { primary care level? } \\
\text { What are the key gaps, barriers, } \\
\text { enabling and reinforcing factors } \\
\text { for PHC facilities to deliver } \\
\text { CMDs prevention and control? }\end{array}$ & $\begin{array}{l}\text { Structured questionnaire } \\
\text { What is the capacity of primary } \\
\text { healthcare facilities in } \\
\text { delivering health services } \\
\text { related to the prevention and } \\
\text { control of CMDs? } \\
\text { In-depth interviews } \\
\text { - What are the gaps, barriers, } \\
\text { enabling and reinforcing factors } \\
\text { in delivering CMDs prevention } \\
\text { and control? }\end{array}$ & $\begin{array}{l}\text { Structured questionnaire } \\
\text { What is the access and } \\
\text { utilization of primary healthcare } \\
\text { services among people with } \\
\text { CMDs? } \\
\text { In-depth interviews } \\
\text { What are factors that influence } \\
\text { their accessing and utilization of } \\
\text { primary healthcare services for } \\
\text { routine chronic disease } \\
\text { management? }\end{array}$ \\
\hline
\end{tabular}

Fig. 1 Overview of research activities and addressed research questions 
Table 1 Overview of the scope of fieldwork research activities

\begin{tabular}{ll}
\hline Level of stakeholders & Activities \\
\hline National Level & $\cdot$ At least 1 individual in-depth interview of a key national level policymaker ${ }^{\text {a }}$ \\
Regional Level (2 regions for each country) & For each region: \\
& $\cdot 2$ individual in-depth interviews of regional level policymakers ${ }^{\text {a }}$ \\
& $\cdot 2$ individual in-depth interviews of district-level policymakers ${ }^{{ }^{a}}$ \\
Primary health care facility level (10 PHC facilities in & For each primary health care facility: \\
total for each country) & $\cdot 1$ Facility Assessment Questionnaire \\
& $\cdot 1$ individual in-depth interview of PHC provider/facility leader ${ }^{\text {b }}$ \\
& $\cdot 10$ structured questionnaires among people with CMDs \\
& $\cdot 1-2$ individual in-depth interview among people with CMDs
\end{tabular}

Note: ${ }^{\text {P}}$ olicymakers include people who hold a position within the district, regional, or national government or an institution that plays a key role in making or implementing policies that relate to the primary health care system or preventing NCDs

${ }^{\mathrm{b}}$ The PHC provider or facility leader needs to be knowledgeable to provide reliable information on behalf of their facility with at least 1 year working experience in the facility, good understanding of the capacity of the facility and NCD services

resource level (below national average development level), diversity in geographical location, and willingness to participate. Five PHC facilities within each region will be selected by local collaborators based on the following four principles: (1) First contact: the selected PHC facilities should be at the grassroots level as the first contact point between the health system and the public. (2) Representativeness: the ten PHC facilities could represent the PHC facilities in resource-limited settings in the country, as in rural areas and urban slums. (3) Feasibility: researchers should be able to travel between PHC facilities under the planned budget. (4) Willingness: PHC facilities are willing to participate in the study and are able to provide reliable information to researchers.

\section{Literature, statistic, and document review}

The purpose of the literature, statistic, and document review is to integrate existing information within our selected countries on (1) the burden and trends of CMDs, and (2) existing and planned national policies and strategies for CMDs prevention and control, focusing on PHC services in resource-poor settings. This information will provide a big picture overview of the challenges and existing strategies in each country and guide us to identify the gaps between the current PHC facility capacity and local needs. The review scope will be limited to national and regional representative information only published after the year 2000. International and national databases will be searched to identify the following types of studies and documents: (1) peer-reviewed literature

Table 2 Basic demographic and economic information of four countries in the study

\begin{tabular}{|c|c|c|c|c|}
\hline & Kenya & Vietnam & China & Nepal \\
\hline \multicolumn{5}{|l|}{ Basic characteristics $^{\text {a }}$} \\
\hline Geographic location & Sub-Saharan Africa & Southeast Asia & East Asia & South Asia \\
\hline Income level & Lower middle & Lower middle & Upper middle & Low \\
\hline GDP at market prices in 2017 (US dollar) & $\$ 79.26$ billion & $\$ 223.78$ billion & $\$ 12.24$ trillion & $\$ 24.88$ billion \\
\hline Population, total in 2017 & 49.70 million & 95.54 million & 1.386 billion & 29.30 billion \\
\hline Life expectancy at birth, total in 2016 (year) & 67.0 & 76.2 & 76.3 & 70.3 \\
\hline Poverty headcount ratio at national poverty lines in 2017 (\% of population) & $36.1 \%$ (in 2015) & $9.8 \%$ (in 2016) & $3.1 \%$ (in 2017) & $25.2 \%$ (in 2010) \\
\hline Human Capital Index & 0.52 & 0.67 & 0.67 & 0.49 \\
\hline \multicolumn{5}{|l|}{ The burden of NCDs and CMDs } \\
\hline Proportional mortality due to NCD (\% of all deaths) ${ }^{\text {b }}$ & $27 \%$ & $77 \%$ & $89 \%$ & $66 \%$ \\
\hline Premature mortality from NCDs between 30 and 70 years (\%) & $13 \%$ & $17 \%$ & $17 \%$ & $22 \%$ \\
\hline Proportional mortality due to Cardiovascular diseases (\% of all deaths) ${ }^{\text {b }}$ & $8 \%$ & $31 \%$ & $43 \%$ & $30 \%$ \\
\hline Proportional mortality due to diabetes (\% of all deaths) ${ }^{\mathrm{b}}$ & $1 \%$ & $4 \%$ & $2 \%$ & $4 \%$ \\
\hline DALYs per 100,000 at all ages due to cardiovascular diseases in $2017^{c}$ & 1967.13 & 4393.08 & 6020.67 & 3859.18 \\
\hline DALYs per 100,000 at all ages due to diabetes in $2017^{c}$ & 630.73 & 1109.21 & 709.68 & 708.95 \\
\hline
\end{tabular}

Notes: ${ }^{a}$ Data from World Bank Data accessed through https://data.worldbank.org/

${ }^{b}$ Data from the World Health Organization, Noncommunicable disease country profiles 2018, accessed

through https://www.who.int/nmh/publications/ncd-profiles-2018/en/

'Global Burden of Disease Collaborative Network. Global Burden of Disease Study 2017 (GBD 2017) Results. Seattle, United States: Institute for Health Metrics and

Evaluation (IHME), 2018. Available from http://ghdx.healthdata.org/gbd-results-tool 
from PubMed, Medline, Cochrane and EMBASE (peerreviewed literature in local languages will also be reviewed if the local database exists and is accessible); (2) national/regional reports and grey literature; and (3) national-level statistics from international organizations or institutions such as the World Bank, WHO, Global Burden of Diseases, etc. Additional file 1 showed the details of the searching strategy for each aim. We will extract data to synthesize on the following information: (1) the disease burden of CMDs in the country; (2) policies related to primary healthcare strengthening or NCDs prevention and management; and (3) national or regional programs delivered through $\mathrm{PHC}$ facilities for promoting the prevention and management of CMDs.

Field activity 1: primary health care facility assessment We will use a structured questionnaire and conduct indepth interviews to assess the capacity of PHC facilities in delivering health services related to the prevention and control of CMDs, as well as identify related gaps, barriers, and enabling and reinforcing factors.

\section{Participant selection}

A facility assessment questionnaire will be administered at each selected PHC facility by the local research team in each country. A knowledgeable PHC health care professional will participate in the study to provide reliable information on behalf of their facility. Participants are eligible if they have been working in the facility for no less than 1 year, have a good understanding of the capacity of the facility and CMD related services, and are willing to participate in the study. We will first contact the leader of the facility as the potential participant. If the leader of the facility does not fulfill the criteria, the leader will recommend an eligible person within the facility to participate in the study.

\section{Instruments development}

The facility assessment questionnaire is developed on the basis of the "WHO sample questionnaire for rapid assessment of capacity in primary care facilities" from the WHO PEN package [10], with revision focusing on CMDs. The questionnaire has five sections: "Equipment \& service availability," "Medicine," "Medical record system," "Service utilization \& referral," and "Financing \& human resources." In addition to the structured questionnaire, the local research team will also conduct semi-structured in-depth interviews among health care professionals. The interview guide consists of nine questions, covering the routine management of CMDs, secondary prevention of CMDs, and the "six building blocks of PHC" (health service delivery, health workforce, health information systems, access to essential medicines, health systems financing, and leadership and governance) [28]. Additional file 2 shows the facility assessment survey and the interview guide.

\section{Field activity 2: patients' surveys and interviews}

Structured questionnaires and in-depth interviews will be conducted to understand the utilization of PHC services among people with CMDs for their routine chronic disease management and to examine the obstacles preventing them from accessing such care in a timely manner.

\section{Participants selection and sample size}

We will apply a stratified convenience sampling approach to recruit participants. Ten CMD patients from each PHC facility (100 in total for each country) will be selected to participate in the structured questionnaire. Eligible participants are adults aged more than 18 years old, who have been diagnosed with heart disease, stroke, hypertension, and diabetes, and are willing to participate and give their consent. To achieve a more representative cohort, the local data collection team will take the following selection criteria into consideration if there are more than ten eligible participants from each facility: five males and five females; at least two participants less than 45 years old; people who have been diagnosed by a health care professional as having heart diseases and stroke will be recruited first; and at least two patients who have not obtained care from PHC facilities in the past year. The recruitment of participants will mainly rely on the staffs from community-based organizations or providers in the PHC facilities. We will conduct informed consent and screening for participant eligibility before administering the survey.

Data collectors will select survey participants to take part in the in-depth interviews if participants are able to communicate well and are willing to share more thoughts. The number of participants involved in the indepth interview will be decided based on a saturation point of obtaining a comprehensive understanding without new information acquired. In general around ten participants from each country are expected.

\section{Instrument design}

The questionnaire for the patient survey is developed based on previous projects $[29,30]$. The questionnaire includes five parts: demographic information and disease history, lifestyle risk factors, routine management, access to essential medicines, and satisfaction towards primary healthcare facilities. The in-depth interview guide aims to explore the gaps, barriers, enabling and reinforcing factors in utilizing the CMDs management and prevention services. It includes four open-ended questions on current routine management of CMDs, needs of CMD prevention and treatment, and comments on PHC services. 
Additional file 3 shows the patients' survey and the interview guide.

\section{Field activity 3: policymaker interviews}

In addition to the interviews of patients and health care professionals, we will conduct in-depth interviews among policymakers at the district, regional, and national level. The aim of these policymaker interviews is to identify the gaps, barriers, and enabling and reinforcing factors for policy and strategy implementation for strengthening primary healthcare.

\section{Participants selection and sample size}

Participants will be identified based on the support from the local collaborators and communities. Participants are eligible if they hold a position within the district, regional, or national government or an institution that plays a key role in making or implementing policies that relate to the PHC system or preventing NCDs.

\section{Instruments design}

The in-depth interviews will be conducted by trained researchers who speak the local languages and understand the local health system. The interview guide consists of open-ended questions covering the aspects of existing policies and action plans related to the prevention and control of NCDs, governance and leadership, factors related to the building blocks of healthcare system such as health financing, human resources, health service delivery, and health information systems that may facilitate the strengthening of the PHC system [28]. Additional file 4 shows the interview guide.

\section{Data collection}

Trained researchers will conduct the structured facility assessment and patient questionnaires through face-toface interviews. If the researchers have language difficulties, a translator with a good understanding of the protocol will be present with the researchers to assist in data collection. The survey will be collected using electronic data collection forms via tablet computers. If the local situation cannot fulfill the basic requirement of electronic data collection, we will adopt a pencil-and-paper approach and the local research team will double-enter the data.

Trained researchers who have experience in conducting qualitative research will conduct the In-depth interviews. Interviews will be conducted in the local language, audio recorded, and then transcribed, verbatim, in local languages for further analysis.

\section{Quality control}

Pilot tests of instruments will be conducted in each country before the data collection to ensure feasibility under the local context. The instruments will be translated from English to the local language and back translated to ensure consistency across countries. Protocol training and technical training among data collectors will also be organized in each country before data collection.

\section{Data analysis and management}

Descriptive analyses will be carried out using STATA statistical software (Version 15, College Station, TX, StataCorp LLC.). The analysis will be performed for each country. Mean and standard deviation will be reported for continuous variables with normal distribution; median and range will be reported if the data is skewed. Frequencies and proportions will be reported for categorical variables. For patients' data, correlation analysis may be performed to explore the associated factors related to the utilization of $\mathrm{PHC}$ services on CMDs management.

For data collected through in-depth interviews, researchers will analyze the transcript in the local language by following a thematic analysis approach [31, 32]. Researchers will first read through all transcripts and then develop themes to capture the important features of the data. Codes will be generated for each transcript line by line and software may be used to facilitate the coding process based on local researchers' capacity and preference. By categorizing the qualitative data into concepts and themes, we will report the key findings on the gaps and barriers of current PHC services on CMD management and prevention.

\section{Discussion}

Strengthening PHC is one of the key strategies to combat the increasing burden of CMDs. With the epidemiological transition occurring in most LMICs, attention has begun to shift to the prevention and control of NCDs, including CMDs [2]. Such a shift poses considerable challenges to the health care system as most LMICs were not prepared for such change [33]. Health care systems have to consider future needs with an integrated approach that could combat the coexistence of persisting infectious diseases and emerging NCDs $[20,21,34]$. As the service-fronting element of the PHC approach, PHC facilities are vital in providing the linkage between the community and the health care system and translating government commitments and public health actions into community-based services [20]. Thus, the contribution of our study is to describe the trends of CMDs' epidemic in within the selected countries, synthesize existing policies and strategies that promote PHC services on prevention and control of CMD, identify gaps in $\mathrm{PHC}$ capacity for implementing existing policy and strategies, and analyze the potential internal 
and external facilitators and barriers that may push forward the strengthening of PHC.

In this study, we will examine PHC facilities from multiple perspectives by using mixed-methods and involving various stakeholders, including patients, health care professionals, and policymakers. The resulting information on disease burden from the literature, statistical, and document review will provide an overview of the epidemic of CMDs in each country with respect to current trends and scope of the challenges. The review on existing policies and programs will provide researchers and stakeholders with an overview of the policy environment and the role of PHC facility within the whole healthcare system. The patient data will focus on factors related to service delivery, with the objective to cast into sharp relief the gaps between available services and the needs of patients. Compared with previous studies, which have solely assessed PHC facilities, our approach is able to provide a more comprehensive view on the current capacity of the grassroots primary health facilities in delivering services for people with CMDs and related internal and external factors. By understanding how grassroots $\mathrm{PHC}$ facilities interact with patients and policies, we will be able to identify potential interventions that are accessible, meet the needs of people with CMDs, can be feasibly delivered by grassroots $\mathrm{PHC}$ facilities in resource-limited settings, and may be sustainably supported by governments.

The focus of LMICs in this study has the potential to inform global efforts in promoting NCDs prevention and control in LMICs. Despite the growing evidence supporting the benefits of PHC-focused health systems in recent decades, there are still gaps in understanding the implementation of a primary care approach for NCD prevention and control in LMICs [35, 36]. On one hand, most LMICs are encountering a more complex situation with regard to the co-existence of communicable and NCDs. On the other hand, fragile health care systems in many LMICs are not adequately prepared for implementing the "best-buy" interventions [36, 37] that have been evaluated in high-income settings. This study has the potential to add new evidence and enlighten LMICs in ways to promote $\mathrm{PHC}$ in a horizontal approach with a specific vertical focus on CMDs.

This study is further unique in that it focuses on resource-limited settings in LMICs. The four countries China, Nepal, Vietnam, and Kenya - share various similarities, notably an increasing burden of CMDs and related risk factors [24-27], and the inequity of health care resources and outcomes within the country [25, 38-40]. They are also distinct, however, with respect to population demographics, healthcare system structure, economic development, and so on. Difficulties in the prevention and control of CMDs may manifest differently across the four countries, but by highlighting the common challenges we can identify potential mitigating strategies in settings where the health workforce, financial input, and medical resources are below each country's average level. In light of such evidence, we will identify potential solutions for implementing the best-buy interventions in resourcelimited settings, which may lead to effective changes in risk factors and health outcomes among more vulnerable populations.

The study is a collaborative effort made by researchers from the four study countries and the results will be delivered to both the international audience and in-country stakeholders. High involvement of local researchers, who are experienced at working effectively within their respective countries, will ensure the effective implementation of the study with full consideration of local context and active involvement of key stakeholders. Local researchers will also be the key persons to deliver the study results to the country stakeholders to expand the study impact. We plan to deliver our study findings as policy briefs to attract a broader international audience. The specific country results will be published as peer-reviewed literature as well as a country-report to the policymakers in the country. Some in-country workshops on study findings will be organized, if feasible, to demonstrate study findings to PHC facilities leaders and discuss the potential action plans for improvement. With these dissemination plans, this project will not only provide evidence to the academic world but also has the potential to bring real-world impact to the local facilities, regions, or broadly.

After forty years since the declaration of Alma Ata [41], which characterized the role of PHC for the first time, researchers and policy makers are still holding our promise in promoting health for all through a PHC approach irrespective of diseases and settings [19, 20, 42]. With the WHO and LMIC governments increasingly committed to equity and universal health coverage, a focus on resource-limited settings in LMICs will bring new opportunities in generating feasible and effective strategies for combating the increasing burden of CMDs, especially among the most vulnerable populations.

\section{Supplementary information}

Supplementary information accompanies this paper at https://doi.org/10. 1186/s41256-019-0124-0.

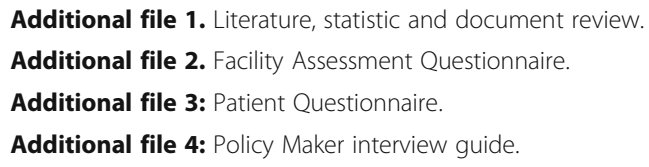

\section{Abbreviations}

CMDs: Cardiometabolic Diseases; LMICs: low- and middle-income countries; NCDs: Non-communicable Diseases; PHC: Primary Health Care; WHO PEN: World Health Organization "Package of Essential NCD interventions for primary care in low-resource settings" 


\section{Acknowledgments}

We would like to thank all researchers who have contributed to the development of the protocol and the coordination and implementation of the study, including Shenglan Tang, Jeffery Moe, Jingyao Zhang, Yang Ying, Heng Jiang, Shree Niwas Khanal, Joshila Rai, Alex Ezeh, Carol Wangui, and Christopher Khayeka. We thank David Hare for his support in proofreading our final draft and made linguistic edits. We would also like to thank the local collaboration institutions: Nepal —-the Institute of Medicine of Tribhuvan University and the Nepal Health Research Council, Vietnam-Hanoi University of Public Health and Kenya-African Population Health \& Research Center.

\section{Authors' contributions}

$E G, S S, X T, N P$ and LLY contributed to the study concept and conceived the study design. BK, CK, TNH, CKW, HVM, TTDH, SK and KG contributed to the development and adaptation of study protocol and instruments in each country. EG drafted the manuscript, HL and SX assisted in the revision of the manuscript. All co-authors contributed to the revision of the manuscript and approved the manuscript for submission.

\section{Funding}

This study is funded by World Health Organization Asia Pacific Observatory on Health System and Policies (to support the activities in Vietnam, China, and Nepal), Duke Kunshan University Education Development Foundation with a philanthropic donation by Yi Ming Foundation and its President, Ms. Guo Tingting (in support of the activities in Kenya). Duke University and Duke Kunshan University provide financial support to students who conducted fieldwork in Vietnam, Nepal, and Kenya for data collection.

\section{Availability of data and materials}

The datasets generated and/or analyzed during the current study are not publicly available but are available from the corresponding author on reasonable request.

\section{Ethics approval and consent to participate}

The study is approved by the Institutional Review Board from Duke Kunshan University in China, and Duke University in the US. The local Institutional Review Board approvals are obtained from Hanoi University of Public Health in Vietnam, the Nepal Health Research Council in Nepal and the African Medical and Research Foundation in Kenya.

\section{Competing interests}

The authors declare that they have no competing interests

\section{Author details}

${ }^{1}$ Global Health Research Center, Duke Kunshan University, No. 8 Duke Avenue, Kunshan 215316, Jiangsu, China. ${ }^{2}$ Global Health Institute, Duke University, Durham, NC, USA. ${ }^{3}$ Department of Psychiatry, George Washington University, Washington, DC, USA. ${ }^{4}$ African Population and Health Research Center, Nairobi, Kenya. ${ }^{5}$ Hanoi University of Public Health, Hanoi, Vietnam.

${ }^{6}$ Transcultural Psychosocial Organization Nepal, Kathmandu, Nepal.

Received: 14 June 2019 Accepted: 10 October 2019

Published online: 12 November 2019

\section{References}

1. Roth GA, Abate D, Abate KH, Abay SM, Abbafati C, Abbasi N, Abbastabar H, Abd-Allah F, Abdela J, Abdelalim A. Global, regional, and national age-sexspecific mortality for 282 causes of death in 195 countries and territories, 1980-2017: a systematic analysis for the global burden of disease study 2017. Lancet. 2018;392(10159):1736-88.

2. GBD 2017 Risk Factor Collaborators. Global, regional, and national comparative risk assessment of 84 behavioural, environmental and occupational, and metabolic risks or clusters of risks for 195 countries and territories, 1990-2017: a systematic analysis for the global burden of disease study 2017. The Lancet. 2018;392(10159):1923-94.

3. Bloom DE, Chisholm D, Jané-Llopis E, Prettner K, Stein A, Feigl A: From burden to "best buys": reducing the economic impact of noncommunicable disease in low-and middle-income countries. In.: Program on the Global Demography of Aging; 2011.
4. Varghese C, Nongkynrih B, Onakpoya I, McCall M, Barkley S, Collins TE. Better health and wellbeing for billion more people: integrating noncommunicable diseases in primary care. Bmj. 2019;364:1327.

5. Mahipala P, Dorji G, Tisocki K, Rani M. A critical review of addressing cardiovascular and other non-communicable diseases through a primary health care approach in the South-East Asia region. Cardiovascular diagnosis and therapy. 2019;9(2):150.

6. Demaio AR, Kragelund Nielsen K, Pinkowski Tersbol B, Kallestrup P, Meyrowitsch DW. Primary health care: a strategic framework for the prevention and control of chronic non-communicable disease. Glob Health Action. 2014;7:24504.

7. Beaglehole R, Epping-Jordan J, Patel V, Chopra M, Ebrahim S, Kidd M, Haines A. Improving the prevention and management of chronic disease in lowincome and middle-income countries: a priority for primary health care. Lancet (London, England). 2008;372(9642):940-9.

8. Ghebreyesus TA, Fore H, Birtanov Y, Jakab Z. Primary health care for the 21 st century, universal health coverage, and the sustainable development goals. Lancet. 2018;392(10156):1371-2.

9. World Health Organization: The world health report 2008: primary health care now more than ever. In.; 2008.

10. World Health Organization: Implementation tools: package of essential noncommunicable (PEN) disease interventions for primary health care in low-resource settings. In.; 2013.

11. Hyon CS, Nam KY, Sun HC, Garg R, Shrestha SM, Ok KU, Kumar R. Package of essential noncommunicable disease (PEN) interventions in primary health-care settings in the Democratic People's Republic of Korea: a feasibility study. WHO South East Asia J Public Health. 2017;6(2):69-73.

12. Organization, $\mathbf{W H}$ : Strengthening health systems to accelerate delivery of noncommunicable diseases services at the primary health care level: one year progress review of the implementation of the 2016 Colombo declaration on NCDs 2017.

13. Mendis S, Al Bashir I, Dissanayake L, Varghese C, Fadhil I, Marhe E, Sambo B, Mehta F, Elsayad H, Sow I. Gaps in capacity in primary care in low-resource settings for implementation of essential noncommunicable disease interventions. Int J Hypertens. 2012;2012:584041.

14. Allen LN, Pullar J, Wickramasinghe KK, Williams J, Roberts N, Mikkelsen B, Varghese C, Townsend N. Evaluation of research on interventions aligned to WHO 'best buys' for NCDs in low-income and lower-middle-income countries: a systematic review from 1990 to 2015. BMJ Glob Health. 2018;3(1):e000535.

15. Mutale W, Bosomprah S, Shankalala P, Mweemba O, Chilengi R, Kapambwe S, Chishimba C, Mukanu M, Chibutu D, Heimburger D. Assessing capacity and readiness to manage NCDs in primary care setting: gaps and opportunities based on adapted WHO PEN tool in Zambia. PLoS One. 2018;13(8):e0200994

16. Rogers HE, Akiteng AR, Mutungi G, Ettinger AS, Schwartz Jl. Capacity of Ugandan public sector health facilities to prevent and control noncommunicable diseases: an assessment based upon WHO-PEN standards. BMC Health Serv Res. 2018;18(1):606.

17. Peck R, Mghamba J, Vanobberghen F, Kavishe B, Rugarabamu V, Smeeth L, Hayes R, Grosskurth H, Kapiga S. Preparedness of Tanzanian health facilities for outpatient primary care of hypertension and diabetes: a cross-sectional survey. Lancet Glob Health. 2014;2(5):e285-92.

18. Nyarko KM, Ameme DK, Ocansey D, Commeh E, Markwei MT, Ohene S-A. Capacity assessment of selected health care facilities for the pilot implementation of package for essential non-communicable diseases (PEN) intervention in Ghana. Pan Afr Med J. 2016;25(Suppl 1):16.

19. Bhutta ZA, Atun R, Ladher N, Abbasi K. Alma Ata and primary healthcare: back to the future. The BMJ. 2018.

20. Hone T, Macinko J, Millett C. Revisiting Alma-Ata: what is the role of primary health care in achieving the sustainable development goals? Lancet. 2018; 392(10156):1461-72

21. Narain J. Integrating services for noncommunicable diseases prevention and control: use of primary health care approach. Indian J Community Med. 2011;36(Suppl1):S67-71.

22. Aryal BK, Daud M, Thapa A, Mahotra A, Magar SA, Malla CK. Assesssment of health facilities for implementation of package of essential noncommunicable disease in Nepal: baseline study in Kailali and Ilam District. J Nepal Health Res Counc. 2018;16(2):149-55.

23. Van Minh H, Do YK, Bautista MAC, Tuan Anh T. Describing the primary care system capacity for the prevention and management of non-communicable diseases in rural Vietnam. Int J Health Plann Manag. 2014;29(2):e159-73.

24. Sharma SR, Page R, Matheson A, Lambrick D, Faulkner J, Mishra SR. Noncommunicable disease prevention in Nepal: systemic challenges and future 
directions. Glob Health Promot. 2017;26:94-7. https://doi.org/10.1177/ 1757975917720800.

25. Achoki T, Miller-Petrie MK, Glenn SD, Kalra N, Lesego A, Gathecha GK, Alam U, Kiarie HW, Maina IW, Adetifa IM. Health disparities across the counties of Kenya and implications for policy makers, 1990-2016: a systematic analysis for the global burden of disease study 2016. Lancet Glob Health. 2019;7(1): e81-95.

26. Zhou M, Wang H, Zhu J, Chen W, Wang L, Liu S, Li Y, Wang L, Liu Y, Yin P. Cause-specific mortality for 240 causes in China during 1990-2013: a systematic subnational analysis for the global burden of disease study 2013. Lancet. 2016;387(10015):251-72

27. Bui TV, Blizzard CL, Luong KN, Truong NLV, Tran BQ, Otahal P, Gall S, Nelson $\mathrm{MR}, \mathrm{Au} \mathrm{TB}, \mathrm{Ha} \mathrm{ST}$, et al. National survey of risk factors for noncommunicable disease in Vietnam: prevalence estimates and an assessment of their validity. BMC Public Health. 2016;16(1):498.

28. World Health Organization: Monitoring the building blocks of health systems: a handbook of indicators and their measurement strategies. In.; 2010.

29. WHO STEPwise approach to chronic disease risk factor surveillance-Instrument [https://www.who.int/hcds/surveillance/steps/STEPS_Instrument_v2.1.pdf].

30. Institute for Health Metrics and Evaluation: Access, Bottlenecks, Costs, and Equity (ABCE). In., vol. 2018; 2011.

31. Castleberry A, Nolen A. Thematic analysis of qualitative research data: is it as easy as it sounds? Currents in Pharmacy Teaching and Learning. 2018;10(6): 807-15.

32. Yin RK. Qualitative research from start to finish: Guilford publications; 2015 .

33. Bollyky TJ, Templin T, Cohen M, Dieleman JL. Lower-income countries that face the most rapid shift in noncommunicable disease burden are also the least prepared. Health Aff. 2017;36(11):1866-75.

34. Kalra S, Akanov ZA, Pleshkova AY. Thoughts, words, action: the Alma-Ata declaration to diabetes care transformation. Diabetes Therapy. 2018;9(3): 873-6.

35. Pradeepa R. The rising burden of diabetes and hypertension in southeast asian and african regions: need for effective strategies for prevention and control in primary health care settings. Int J Hypertens. 2013;2013:409083.

36. Samb B, Desai N, Nishtar S, Mendis S, Bekedam H, Wright A, Hsu J, Martiniuk A, Celletti F, Patel K, et al. Prevention and management of chronic disease: a litmus test for health-systems strengthening in low-income and middleincome countries. Lancet. 2010;376(9754):1785-97.

37. World Health Organization Maximizing Positive Synergies Collaborative Group. An assessment of interactions between global health initiatives and country health systems. Lancet. 2009;373(9681):2137-69.

38. Duong DB, Minh HV, Ngo LH, Ellner AL. Readiness, availability and utilization of rural Vietnamese health facilities for community based primary Care of non-communicable Diseases: a CrossSectional survey of 3 provinces in northern Vietnam. Int J Health Policy Manag. 2018:8(3):150-7.

39. Tang S, Meng Q, Chen L, Bekedam H, Evans T, Whitehead M. Tackling the challenges to health equity in China. Lancet. 2008:372(9648):1493-501.

40. Haregu TN, Oti S, Egondi T, Kyobutungi C. Co-occurrence of behavioral risk factors of common non-communicable diseases among urban slum dwellers in Nairobi, Kenya. Glob Health Action. 2015;8(1):28697.

41. World Health Organization: Declaration of Alma Ata. In: International conference on primany health care. Alma-Ata: World Health Organization; 1978.

42. Rawaf S, De Maeseneer J, Starfield B. From Alma-Ata to Almaty: a new start for primary health care. Lancet. 2008;372(9647):1365-7.

Ready to submit your research? Choose BMC and benefit from:

- fast, convenient online submission

- thorough peer review by experienced researchers in your field

- rapid publication on acceptance

- support for research data, including large and complex data types

- gold Open Access which fosters wider collaboration and increased citations

- maximum visibility for your research: over $100 \mathrm{M}$ website views per year

At BMC, research is always in progress.

Learn more biomedcentral.com/submissions 